\title{
Comparison of a salt check sheet with 24-h urinary salt excretion measurement in local residents
}

\author{
Kenichiro Yasutake ${ }^{1}$, Emiko Miyoshi ${ }^{1}$, Tomomi Kajiyama ${ }^{1}$, Yoko Umeki ${ }^{2}$, Yukiko Misumi ${ }^{2}$, Noriko Horita ${ }^{2}$, \\ Yusuke Murata ${ }^{3}$, Kenji Ohe ${ }^{3}$, Munechika Enjoji ${ }^{3}$ and Takuya Tsuchihashi ${ }^{4}$
}

The salt check sheet developed by Tsuchihashi et al. is widely used in general practice to assess salt intake and the associated diets. However, its appropriateness for the general population has not been assessed alongside 24-h urinary salt excretion monitoring. Therefore, in local residents, we analyzed the correlation between check-sheet scores and 24-h urinary salt excretion levels to determine the appropriateness of the check sheet. We asked 176 local residents to complete the salt check sheet and provide urinary samples; the latter were obtained using a proportional sampling method over a 24-h period. One hundred and forty subjects completed the study (men/women: $23 / 117$, mean \pm s.d. age: $52.7 \pm 19.6$ years, blood pressure: $122.3 \pm 18.0 / 74.3 \pm 11.1 \mathrm{~mm} \mathrm{Hg}$ ), of whom $51(36.4 \%)$ had hypertension. The total salt check-sheet scores were widely distributed (mean \pm s.d.: $11.1 \pm 4.2$ points, range: $0-22$ points), and the subjects were divided into the following groups on the basis of salt levels: $29.3 \%$ were 'low' (0-8 points), $42.8 \%$ were 'medium' (9-13 points), $23.6 \%$ were 'high' (14-19 points) and $4.3 \%$ were 'very high' ( $>20$ points). The mean 24 -h urinary salt excretion level was $8.5 \pm 3.3 \mathrm{~g}$. The subjects with higher salt-intake levels tended to have increased 24-h urinary salt excretion levels, with significant differences between the three groups ('low' vs. 'medium' vs. 'high to very high' salt levels: $7.6 \pm 2.9 \mathrm{~g}$ vs. $8.4 \pm 2.8 \mathrm{~g}$ vs. $9.6 \pm 4.2 \mathrm{~g}$, respectively; $P=0.03$ ). The total salt check-sheet scores significantly correlated with the 24-h urinary salt excretion levels $(r=0.27 ; P<0.01)$. Thus, the salt check sheet is applicable for the general population. Hypertension Research (2016) 39, 879-885; doi:10.1038/hr.2016.79; published online 7 July 2016

Keywords: salt check sheet; salt intake; salt restriction; screening tool; urinary salt excretion

\section{INTRODUCTION}

At the UN High Level meeting on Non-communicable Diseases in 2011, five priority interventions were identified, and salt reduction was selected as the second most important. ${ }^{1}$ This decision was based on the rate at which low salt intake decreases blood pressure, which in turn attenuates cerebral vascular disease and myocardial infarction. ${ }^{2}$ In the Japanese population, the death rate related to various risk factors shows that smoking is the most important risk factor, followed by hypertension; excessive salt intake ranks fifth on this scale. ${ }^{3}$ This shows that low salt intake is an extremely important aspect of health. However, the salt intake of Japanese people is higher than that of the populations of other countries. ${ }^{4}$ Moreover, even though patients with hypertension or cardiovascular disease should severely restrict their salt intake, they consume more salt on average than those without these conditions, ${ }^{5}$ and this excessive salt intake has not lessened over time. $^{6}$

In the effort to lower salt intake nationwide, advertisements and mass media campaigns are an effective strategy. Nonetheless, it remains difficult to motivate people toward this desired end on a national scale. ${ }^{1}$ In addition, consumers often confuse the terms 'sodium content' and 'salt content' on food packages, ${ }^{7}$ and are thus ill informed to make decisions regarding their salt intake. The salt content of many processed foods and ready-made dishes in Japan is not evident in their taste, making it difficult for individuals to determine how much salt they consume daily. In 2013, Tsuchihashi et al. ${ }^{8}$ developed a salt check sheet (Table 1), whereby examinees and examiners can easily evaluate salt intake, as well as identify the foods responsible for this intake. The evaluation can be carried out routinely at local clinics. The salt checklist comprises a single A4 sheet with 13 questions about daily salt intake. It takes $3-5 \mathrm{~min}$ to complete, making it practical to use. Furthermore, a previous study involving 270 outpatients with hypertension found a significant correlation between the check-sheet scores and urinary salt levels assessed using on-the-spot urine tests; the correlation was calculated using the Tanaka equation ${ }^{9}(r=0.30 ; P<0.01) .{ }^{8}$ However, this report only examined outpatients with hypertension. Therefore, it is unclear

\footnotetext{
${ }^{1}$ Department of Nutritional Sciences, Faculty of Nutritional Sciences, Nakamura Gakuen University, Fukuoka, Japan; ${ }^{2}$ Department of Health and Nutrition Sciences, Faculty of Health and Nutrition Sciences, Nishikyushu University, Saga, Japan; ${ }^{3}$ Health Care Center, Fukuoka University, Fukuoka, Japan and ${ }^{4}$ Hypertension Center, Steel Memorial Yawata Hospital, Kitakyushu, Japan

Correspondence: Dr K Yasutake, Department of Nutritional Sciences, Faculty of Nutritional Sciences, Nakamura Gakuen University, 5-7-1 Befu, Jonan-ku, Fukuoka 814-0198, Japan.

E-mail: yasutake@nakamura-u.ac.jp

Received 6 January 2016; revised 2 May 2016; accepted 20 May 2016; published online 7 July 2016
} 
Table 1 Salt check sheet. Your salt check sheet, circle what fits you and write the total score at the end

\begin{tabular}{|c|c|c|c|c|c|}
\hline & & 3 points & 2 points & 1 point & 0 \\
\hline \multirow{7}{*}{ 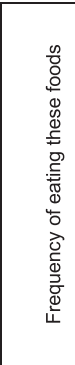 } & miso (fermented soybean paste) soup, soup, etc. & $\begin{array}{l}\text { more than } 2 \text { bowls } \\
\text { a day }\end{array}$ & $\begin{array}{l}\text { about } 1 \text { bowl } \\
\text { a day }\end{array}$ & $\begin{array}{l}\text { two-three bowls } \\
\text { a week }\end{array}$ & hardly eat \\
\hline & pickles, pickled plums, etc. & $\begin{array}{l}\text { more than twice } \\
\text { a day }\end{array}$ & $\begin{array}{l}\text { about once } \\
\text { a day }\end{array}$ & $\begin{array}{l}\text { two-three times } \\
\text { a week }\end{array}$ & hardly eat \\
\hline & $\begin{array}{l}\text { fish-paste products such as chikuwa (tubular fish sausage) and kamaboko } \\
\text { (steamed fish paste) }\end{array}$ & & eat frequently & $\begin{array}{l}\text { two-three times } \\
\quad \text { a week }\end{array}$ & hardly eat \\
\hline & $\begin{array}{l}\text { horse mackerel cut open lengthwise and dried, dried fish seasoned with mirin } \\
\text { (sweetened alcohol for use in cooking), salted salmon, etc. }\end{array}$ & & eat frequently & $\begin{array}{l}\text { two-three times } \\
\text { a week }\end{array}$ & hardly eat \\
\hline & ham or sausage & & eat frequently & $\begin{array}{l}\text { two-three times } \\
\text { a week }\end{array}$ & hardly eat \\
\hline & $\begin{array}{l}\text { noodles such as udon (Japanese wheat noodles) and } \\
\text { ramen (Japanese-style Chinese noodles) }\end{array}$ & almost every day & $\begin{array}{c}2 \text { or } 3 \text { bowls } \\
\text { a week }\end{array}$ & less than once a week & don't eat \\
\hline & $\begin{array}{l}\text { senbei (Japanese crackers), okaki (thinly-cut and dried rice cakes), potato } \\
\text { chips, etc. }\end{array}$ & & frequently & $\begin{array}{l}\text { two-three times } \\
\text { a week }\end{array}$ & hardly eat \\
\hline \multicolumn{2}{|c|}{ How frequent do you season with soy sauce or other sauces? } & $\begin{array}{l}\text { season frequently } \\
\text { (almost every meal) }\end{array}$ & once a day & season sometimes & don't season \\
\hline \multicolumn{2}{|c|}{ How much udon, ramen, or other soups do you consume? } & entire bowl & about half & some & little \\
\hline \multicolumn{2}{|c|}{ Do you eat out or have convenience-store-bought bento (lunch plate) for lunch? } & almost every day & $\begin{array}{l}\text { about } 3 \text { times } \\
\text { a week }\end{array}$ & $\begin{array}{c}\text { about once } \\
\text { a week }\end{array}$ & no \\
\hline \multicolumn{2}{|c|}{ Do you eat out or have ready-made side dishes for dinner? } & almost every day & $\begin{array}{c}\text { about } 3 \text { times } \\
\text { a week }\end{array}$ & $\begin{array}{l}\text { about once } \\
\text { a week }\end{array}$ & no \\
\hline \multicolumn{2}{|c|}{ How salty are your home-made dishes compared with those you eat out? } & heavy & same & & light \\
\hline \multicolumn{2}{|c|}{ Do you think you eat a lot? } & more than others & & same as others & less than others \\
\hline \multicolumn{2}{|r|}{ total score of the items you circled } & 3 points $\times$ & 2 points $\times$ & 1 point $\times$ & 0 point $\times$ \\
\hline \multicolumn{2}{|r|}{ subtotal } & $\ldots$ points & $\ldots$ points & $\ldots$ points & _ points \\
\hline \multicolumn{2}{|r|}{ total points } & \multicolumn{4}{|r|}{$\ldots$ points } \\
\hline
\end{tabular}

\begin{tabular}{|c|c|l|}
\hline $\begin{array}{c}\text { check } \\
\text { below } \square\end{array}$ & total points & evaluation \\
\hline$\square$ & $0-8$ & You are not taking excess salt. Maintain this diet for salt restriction. \\
\hline$\square$ & $9-13$ & Your salt intake is average. Start a little stricter salt restriction. \\
\hline$\square$ & $14-19$ & You have excessive salt intake. You need to lower your salt intake by changing your diet salt content and eating behavior. \\
\hline$\square$ & 20以上 & You have too much salt intake. You need to totally change your diet salt content and eating behavior. \\
\hline
\end{tabular}

whether the results can be applied to the general population; furthermore, the study did not assess the usefulness of measuring urinary salt excretion in 24-h urine samples. The aim of this study was to evaluate the validity of the salt check sheet by analyzing the correlation between the total salt check-sheet scores and 24-h urinary salt excretion levels in the local general population.

\section{METHODS}

\section{Subjects}

One hundred and seventy-six participants (men/women: 27/149) were selected from among 222 applicants on the basis of the following inclusion criteria: (1) residence in the Saga or Fukuoka prefectures (local), (2) age between 20 and 80 years, (3) attendance at the research briefing and (4) agreement to participate in the study. The exclusion criteria were (1) physical, psychological or social difficulties that burdened the daily life of the applicant, (2) lack of personal autonomy and (3) kidney disease that was currently being treated with diuretics. The study protocol adhered to the ethical guidelines of the 1975 Declaration of Helsinki and was approved by Nishikyushu University, and written informed consent for voluntary participation was obtained from each subject (Nishikyushu University H24-4).

\section{Intervention schedule}

This study was conducted in the Saga prefecture in March and April 2014, and in the Fukuoka prefecture in October and November 2015. The subjects were asked to complete the salt check sheet on the day they gave consent. Within
2 weeks, on a convenient day, the subjects 24 -h urine salt levels were measured. In women, this measurement was conducted on a non-menstrual day. The participants measured their own blood pressure on the first and last days of urine collection. The subjects were asked to maintain their usual diet during the urine collection period, without special restrictions.

\section{Salt check sheet}

The salt check sheet is a single A4 sheet with 13 questions that take 3-5 min to answer (Table 1). The 13 questions can be categorized as follows: seven items evaluate the intake of salty meals, such as miso soup, pickles and noodles; four evaluate the use of salty sauces (that is, soy sauce), eating out, and home-meal replacement; and two evaluate the seasoning content and size of homemade meals. Each question is scored up to three points for a total of 35 points, and salt content is characterized as low (0-8 points), medium (9-13 points), high (14-19 points) or very high ( $>20$ points). ${ }^{8}$ The check sheet was designed on the basis of the relationship between salt intake (estimated using a BDHQ (brief-type, self-administered, diet history questionnaire) $)^{10,11}$ and 24-h urinary salt excretion in outpatients with hypertension in Fukuoka prefecture. ${ }^{12}$

\section{Twenty-four-hour urine collection}

Twenty-four-hour urine was collected via a proportional sampling method using a partition cup, which collects $1 / 50$ of the total 24 -h urine volume. ${ }^{13}$ One previous study showed a high correlation between the partition cup and total-volume methods in terms of both urine volume $(r=0.97)$ and salt excretion $(r=0.98) .{ }^{13}$ Twenty-four-hour urine was defined as the total volume 
of urine starting with the second morning urine (after discarding the first one at 0600 hours) and ending with the first morning urine of the next day. The precise start and end times for the urine collections were reported by each subject. ${ }^{14,15}$ The collected urine sample was recovered thereafter for analysis. The total urine volume, as well as urine creatinine, sodium and potassium levels, was analyzed by SRL (Tokyo, Japan). Creatinine levels were analyzed using an enzyme-based method, and sodium and potassium levels were analyzed using an electrode-based method. When the 24-h urinary creatinine levels were within $\pm 30 \%$ of the predicted value, urine collection was considered successful; all other samples were excluded. ${ }^{14-17}$

\section{Blood pressure}

The participants measured their blood pressure, after resting for $5 \mathrm{~min}$, using a digital, upper arm, automatic blood pressure-measuring instrument (HEM-7080 IC; Omron, Kyoto, Japan). They did so at the beginning and end of the 24-h urine collection period-immediately after the early morning urination. The average of two consecutive measurements was recorded. We diagnosed patients as having high blood pressure if (1) they had been prescribed antihypertensive drugs or (2) they had a systolic blood pressure of $>135 \mathrm{~mm} \mathrm{Hg}$ or a diastolic blood pressure of $>85 \mathrm{~mm} \mathrm{Hg}$.

\section{Table 2 Characteristics of the study subjects}

\begin{tabular}{lc}
\hline Number & 140 \\
Sex (male/female) & $23 / 117$ \\
Age (years) & $52.7 \pm 19.6$ \\
BMI & $22.5 \pm 3.2$ \\
SBP & $122.3 \pm 18.0$ \\
DBP & $74.3 \pm 11.1$ \\
Obesity & $24(17.1 \%)$ \\
Hypertension & $51(36.4 \%)$ \\
Antihypertensive drugs (other than diuretics) & $25(17.8 \%)$ \\
Smoker/past smoker/nonsmoker & $6 / 17 / 117(4.3 \% / 12.1 \% / 83.6 \%)$
\end{tabular}

Abbreviations: BMI, body mass index; DBP, diastolic blood pressure; SBP, systolic blood pressure

Data expressed as number (\%) or mean \pm s.d.

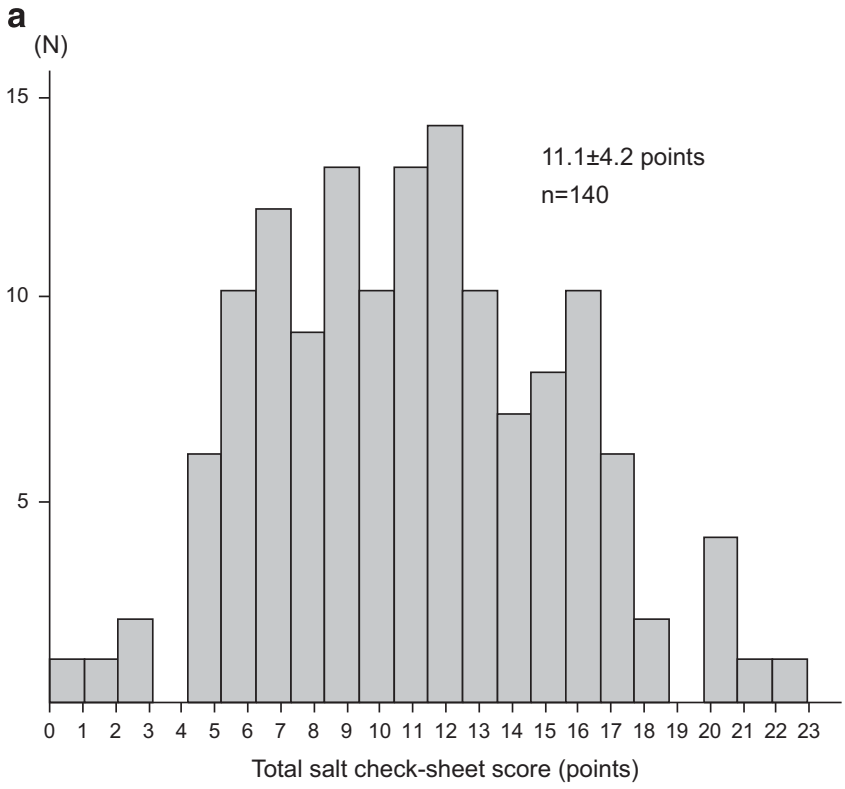

\section{Statistical analysis}

Statistical data were presented as the mean \pm s.d. The paired $t$-test was used to compare two groups, and a variance analysis was used to compare three or more groups. When the results were significant, the Tukey-Kramer test was used for post hoc analysis. The Pearson product-moment correlation coefficient was used to analyze the correlation between groups. The significance level was set at $P<0.05$. Finally, JMP10 statistical software (SAS Institute, Cary, NC, USA) was used for the statistical analyses.

\section{RESULTS}

Of the 176 original participants, 140 subjects were ultimately included in the study; five were excluded because they withdrew their consent and 31 failed to collect the $24-h$ urine samples. The subjects characteristics were as follows: the number of men/women was 23/117, mean age was $52.7 \pm 19.6$ years, mean body mass index (BMI) was $22.5 \pm 3.2 \mathrm{~kg} \mathrm{~m}^{-2}$ and mean self-measured blood pressure was $122.3 \pm 18.0 / 74.3 \pm 11.1 \mathrm{~mm} \mathrm{Hg} ; 17.1 \%$ of the subjects were obese (BMI $\left.>25 \mathrm{~kg} \mathrm{~m}^{-2}\right)$. Fifty-one subjects $(36.4 \%)$ had hypertension, 25 $(17.8 \%)$ of whom were taking antihypertensive drugs other than diuretics (Table 2).

The mean total salt check-sheet score was $11.1 \pm 4.2$ points (range: 0-22 points), and the scores were widely distributed among the subjects, from 'low' to 'very high' (Figure 1a). We assigned 41 subjects $(29.3 \%)$ to the 'low salt' group (0-8 points), 60 (42.8\%) to the 'medium salt' group (9-13 points), 33 (23.6\%) to the 'high salt' group (14-19 points) and $6(4.3 \%)$ to the 'very high salt' group ( $>20$ points). Meanwhile, the $24-\mathrm{h}$ urine collection showed a mean daily urinary salt excretion level of $8.5 \pm 3.3 \mathrm{~g}$ (Figure $1 \mathrm{~b}$ ).

We compared the 'low,' 'medium' and 'high-to-very high' salt groups in terms of urinary salt excretion levels, and found that the 24-h urinary salt excretion level tended to increase as salt intake increased; there was a significant difference between the three groups in this regard ('low'vs. 'medium' vs. 'high-to-very high' groups: $7.6 \pm 2.9$ g vs. $8.4 \pm 2.8$ g vs. $9.6 \pm 4.2 \mathrm{~g} ; P=0.03)$.

Furthermore, the post hoc test showed a significant difference between the 'low' and 'high-to-very high' groups $(P=0.02$; Figure 2). Notably, no significant difference was observed between

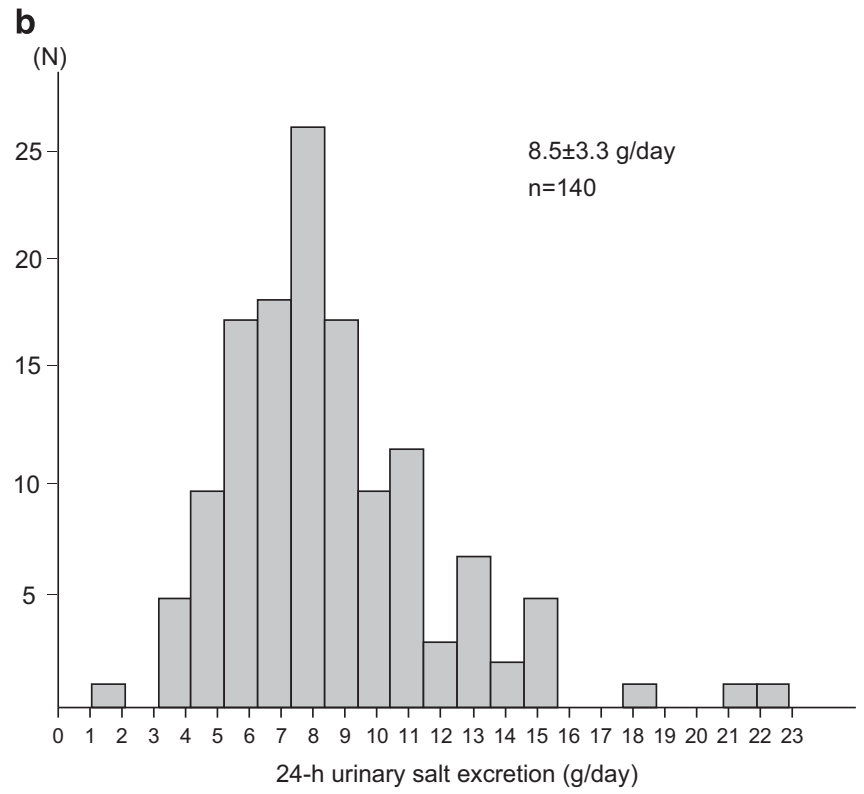

Figure 1 (a) Distribution of total salt check-sheet scores. (b) Distribution of 24-h salt excretion. 
subjects with hypertension and those without ('non-hypertensive' $v$ s. 'hypertensive' groups: $8.4 \pm 3.0 \mathrm{~g}$ per day $(n=89)$ vs. $8.7 \pm 3.8 \mathrm{~g}$ per day $(n=51) ; P=0.71)$.

The salt check-sheet score was significantly positively correlated with the 24-h urinary salt excretion level $(r=0.27 ; P<0.01$; Figure 3$)$. In addition, the total salt check-sheet score was significantly correlated with 24 -h urinary salt excretion levels, in both the ' $\leqslant 65$ years old' age group $(r=0.22 ; P=0.04 ; n=88)$ and the ' $>65$ years old' age group $(r=0.34 ; P=0.02 ; n=48)$. The correlation was also significant in both the 'non-hypertensive' group $(r=0.22 ; P=0.04 ; n=89)$ and the 'hypertensive' group $(r=0.34 ; P=0.01 ; n=51)$. A sex-based analysis revealed a significant positive correlation between the total salt check-sheet score and 24-h urinary salt excretion levels in women $(r=0.19 ; P=0.04 ; n=113)$, but not in men $(r=0.30 ; P=0.16$; $n=23)$. A significant positive correlation occurred in the obese group $(r=0.42 ; P=0.04 ; n=24)$, but not in the nonobese group $(r=0.15$; $P=0.11 ; n=116)$ between the total salt check-sheet score and 24 -h urinary salt excretion level.

We compared the total salt check-sheet scores within various descriptive categories: sex, age ( $\leqslant 65$ years or $>65$ years), obesity (with or without) and hypertension (with or without). Concerning sex, the total salt check-sheet score was higher in men than in women $(13.3 \pm 4.1$ vs. $8.1 \pm 3.1 ; P=0.01)$. An especially significant difference was found between the sexes regarding the intake of salty food $(P=0.01)$, as well as seasoning of homemade meals and eating out/home-meal replacement $(P=0.01)$. With regard to age, the total salt check-sheet scores were significantly higher in the ' $\leqslant 65$ years old' group than in the ' $>65$ years old' group $(11.8 \pm 4.2$ vs. $9.8 \pm 4.1$; $P=0.01$ ). An especially significant difference was found between the age groups in terms of seasoning home meals $(P=0.00)$, eating out/home-meal replacement, and seasoning and meal amount of food $(P=0.00)$. The total salt check-sheet score was higher in obese subjects than in nonobese subjects $(10.6 \pm 4.2$ vs. $13.6 \pm 3.5 ; P=0.00)$. This difference was especially pronounced with regard to the frequency with which high-salt foods were consumed $(P=0.01)$, as well as to the seasoning and size of homemade dishes $(P=0.01)$. Conversely, subjects with hypertension did not differ from

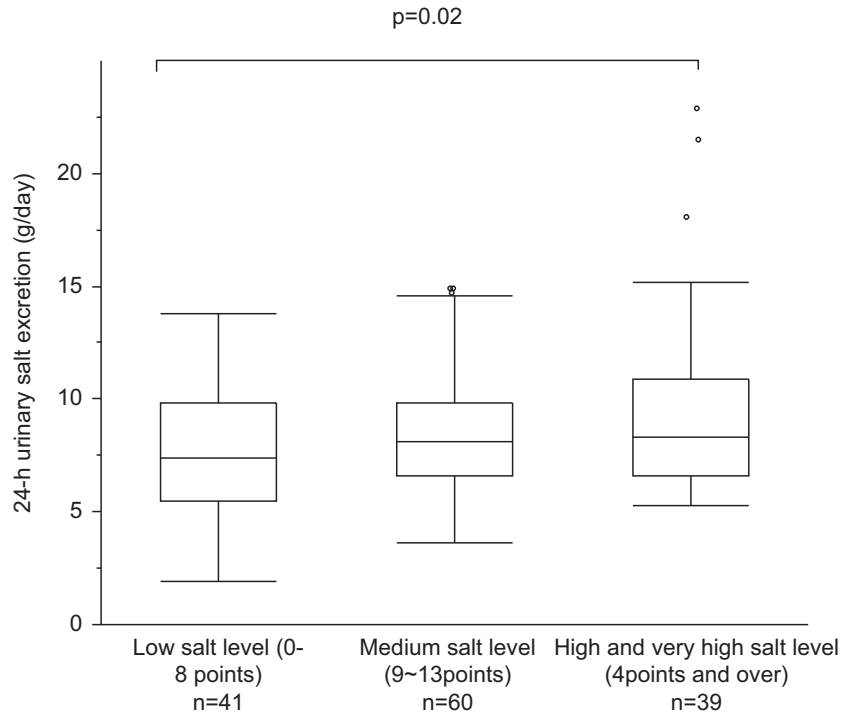

Figure 2 Relationship between salt-intake levels evaluated using the salt check sheet and 24-h salt excretion level. those without in terms of the total salt check-sheet score or the aforementioned descriptive categories (Table 3).

\section{DISCUSSION}

This study validated the salt check sheet by comparing it with 24 -h urinary salt excretion levels in local residents. A significant correlation was found between the total salt check-sheet score and the 24-h urinary salt excretion level (Figures 2 and 3), suggesting that the salt intake of the general population can be screened using the salt check sheet. Moreover, the check sheet not only screens for salt-intake level, but also identifies the food and diet types that lead to the salt intake. Indeed, our results identified the food types that led to salt intake in terms of sex, age and obesity (Table 3 ).

However, three points must be considered at this stage. First, in our study, the mean 24-h urinary salt excretion levels were $8.5 \pm 3.3 \mathrm{~g}$, which is lower than the mean salt intake of the general Japanese population. ${ }^{4,6}$ This might have influenced the results such that subjects with hypertension did not differ from those without in terms of either 24-h urinary salt excretion levels or salt check-sheet scores. However, previous research into the validity of the salt check sheet in hypertensive cases yielded a daily salt excretion level of $8.9 \mathrm{~g},{ }^{8}$ which is comparable with that of our population, and generated similar results.

Second, when analyzed on the basis of sex, the total check-sheet scores and 24-h urinary salt excretion levels did not show any significant correlation, perhaps because of the low number of men. A significant correlation between check-sheet score and 24-h urinary salt excretion levels was shown in obese subjects, but not in nonobese subjects. Perhaps the result in nonobese subjects was derived from over or understatement of salt intake. Over and understatement is common in dietary questionnaires, and BMI is the factor most associated with statement error. ${ }^{18}$ Murakami et al. ${ }^{19}$ reported that, even in the nonobese (BMI $<25 \mathrm{~kg} \mathrm{~m}^{-2}$ ) group, subjects with lower BMI tended to state intakes that were higher than reality, and those with higher BMI tended to do the opposite. Therefore, in regard to BMI, the possibility of over and understatements should be considered in the context of the salt check sheet.

Third, with regard to the comparison of the 'low,' 'medium' and 'high-to-very high' salt groups, which were divided on the basis of the salt check-sheet scores, there were no significant differences in 24 -h

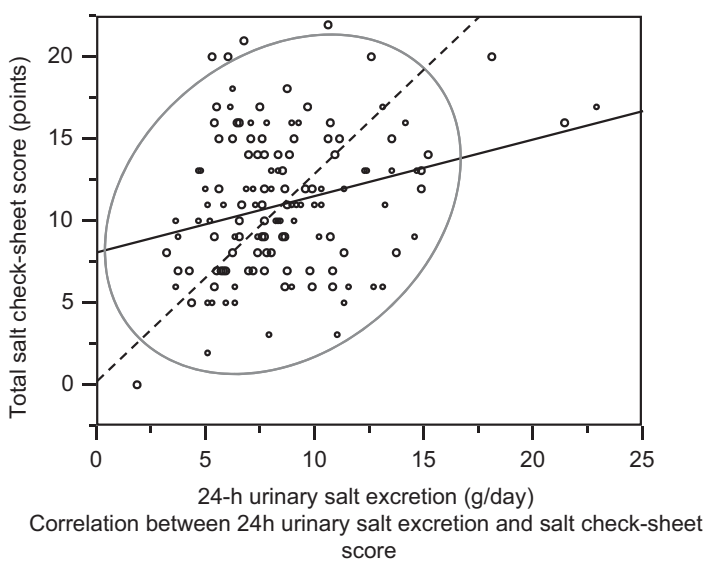

Figure 3 Significant positive correlation between salt check-sheet scores and 24-h salt excretion levels. The dashed line is the identity line, and the solid line is the regression line (total salt check-sheet scores $=8.157662$ $+0.3438676 \times 24-h$ urinary salt excretion). The circle is a normal density ellipse $(P=0.95)$ enclosing the two variables. 
Table 3 Comparison of the scores in the salt check-sheet items on sex, age, obesity and diagnosis of hypertension

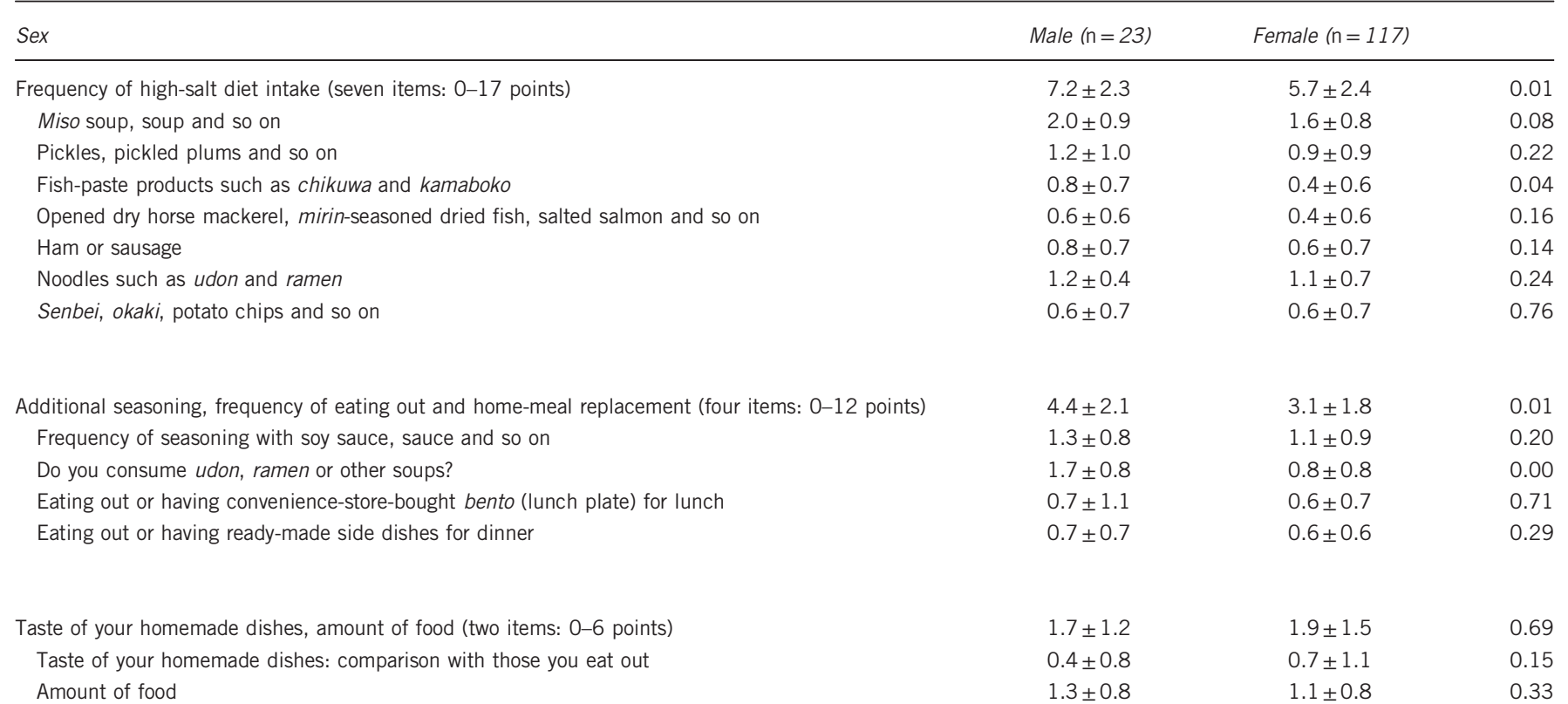

Pickles, pickled plums and so on

Fish-paste products such as chikuwa and kamaboko

$0.5 \pm 0.6$

$1.7 \pm 0.8$

Opened dry horse mackerel, mirin-seasoned dried fish, salted salmon and so on

$0.4 \pm 0.6$

$1.1 \pm 1.0$

$0.7 \pm 0.7$

$0.4 \pm 0.6$

0.28

Noodles such as udon and ramen

$1.1 \pm 0.6$

$0.5 \pm 0.7$

0.46

Senbei, okaki, potato chips and so on

$0.7 \pm 0.7$

0.14

$0.5 \pm 0.6$

0.55

$3.7 \pm 2.1$

$1.2 \pm 0.9$

$2.7 \pm 1.5$

0.00

Additional seasoning, frequency of eating out and home-me
Frequency of seasoning with soy sauce, sauce and so on

$1.1 \pm 0.8$

$1.0 \pm 0.8$

0.24

Do you consume udon, ramen or other soups?

$0.7 \pm 0.9$

$0.7 \pm 0.8$

0.01

Eating out or having convenience-store-bought bento (lunch plate) for lunch

$0.7 \pm 0.7$

$0.4 \pm 0.6$

0.08

Eating out or having ready-made side dishes for dinner

\section{$2.1 \pm 1.5$}

$0.8 \pm 1.1$

$0.5 \pm 0.6$

0.06

Taste of your homemade dishes, amount of food (four items: 0-6 points)

Taste of your homemade dishes: comparison with those you eat out

$1.3+0.8$

$1.3 \pm 1.2$

0.00

Amount of food

$11.8 \pm 4.2$

$0.4 \pm 0.9$

0.02

$0.9 \pm 0.7$

0.00

Total

Without $(\mathrm{n}=116)$

$9.8 \pm 4.0$

0.01

Obesity

$5.7 \pm 2.4$

$1.6 \pm 0.8$

Miso soup, soup and so on

Pickles, pickled plums and so on

Fish-paste products such as chikuwa and kamaboko

Opened dry horse mackerel, mirin-seasoned dried fish, salted salmon and so on

Ham or sausage

Noodles such as udon and ramen

Senbei, okaki, potato chips and so on

Additional seasoning, frequency of eating out and home-meal replacement (four items: 0-12 points)

Frequency of seasoning with soy sauce, sauces and so on
$0.9 \pm 0.9$

$0.4 \pm 0.6$

$0.4 \pm 0.6$

$0.6 \pm 0.7$

$1.1 \pm 0.7$

$0.6 \pm 0.7$

$3.2 \pm 2.0$
$1.1 \pm 0.8$

With $(n=24)$

$\begin{array}{ll}7.0 \pm 1.9 & 0.01 \\ 1.8 \pm 0.7 & 0.53 \\ 1.2 \pm 0.9 & 0.21 \\ 0.7 \pm 0.7 & 0.09 \\ 0.5 \pm 0.6 & 0.63 \\ 0.9 \pm 0.6 & 0.05 \\ 1.3 \pm 0.6 & 0.15 \\ 0.6 \pm 0.6 & 0.89 \\ & \\ 3.9 \pm 1.7 & 0.07 \\ 1.3 \pm 0.9 & 0.53\end{array}$




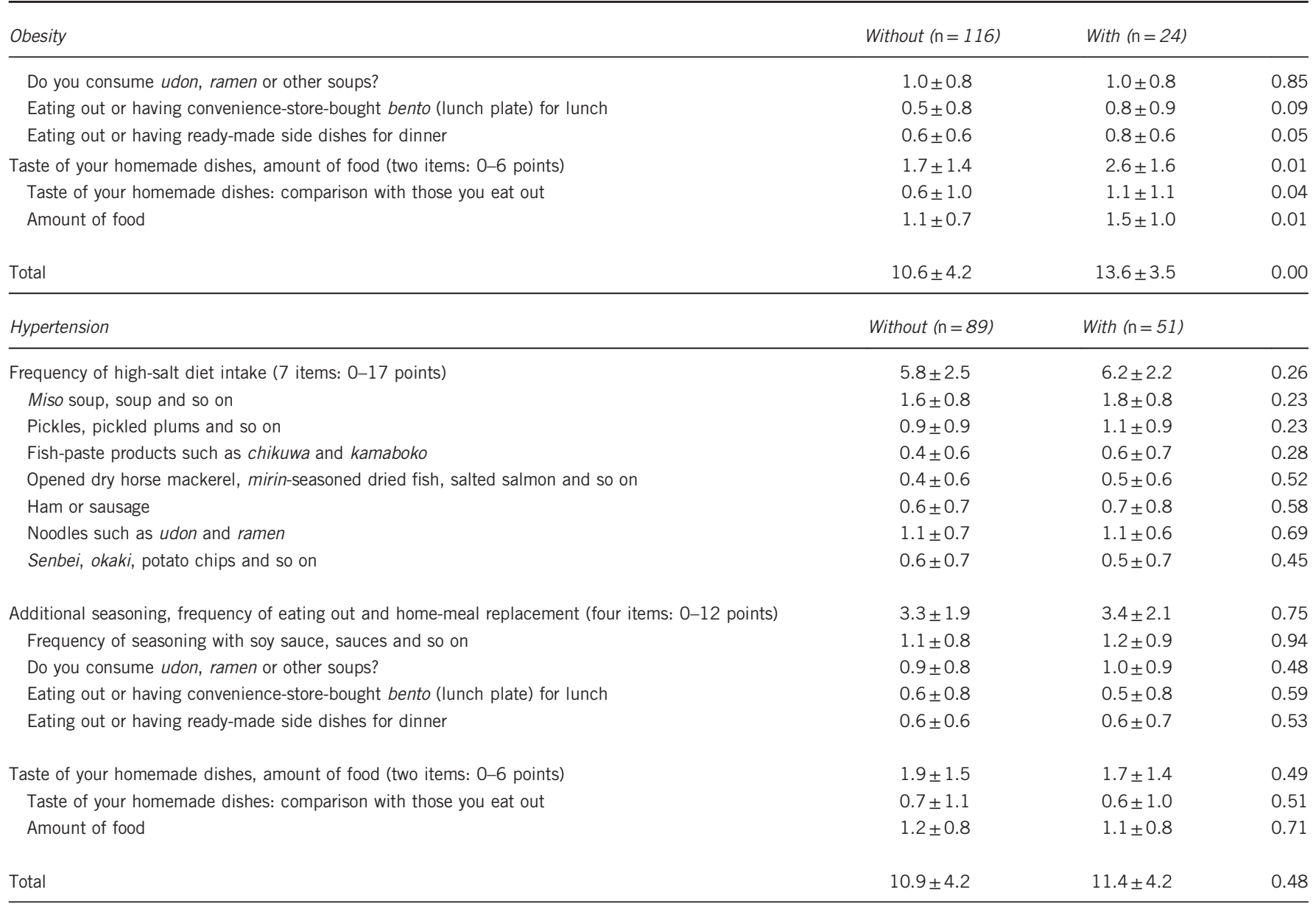

urinary salt excretion levels between the 'low' and 'medium' groups or between the 'medium' and 'high-to-very high' groups. The correlation coefficient between total salt check-sheet scores and 24-h urinary salt excretion levels was low (0.27). However, the 24-h urinary salt excretion levels reflect the salt intake on a specific day, whereas the salt check sheet assesses the average daily salt intake. Thus, the two assessment methods are unlikely to correlate strongly in any case. Our results are compatible with this argument. Indeed, one previous study reported that the correlation coefficient between the results of the BDHQ, ${ }^{10,11}$ which assesses participants' daily eating behavior over the previous month, and those of 24-h urinary salt excretion measurement was 0.34 -comparable with the coefficient in our study. $^{12}$

The questions on the salt check sheet address a high-salt diet and eating behavior, making it less time consuming and easier to complete than the BDHQ specified above. ${ }^{10,11}$ When focusing on salt restriction in the population, it is more important to limit individual salt intake than to compare the accuracies of salt-intake estimates. The salt check sheet is efficient in terms of time; it confers low psychological and physical burdens on subjects and instructors, and it can be repeated frequently. Thus, it can be used to inform the public about salt intake, and thus motivate individuals to monitor their own diets in this regard.

One limitation of the present research was the small number of men: only $16.4 \%$. We need to reevaluate the measured parameters among men in the local community. Second, this study used the data from 24-h urinary salt excretion measurements on one specific day. Thus, to assess longer-term fluctuations in salt intake, ${ }^{20}$ we would need to evaluate the same parameters over longer periods. Third, because the salt check sheet was based on nutritional data from people in a small local area, ${ }^{8,12}$ the relationship between the check sheet and 24-h urine salt excretion should be analyzed in different subjects from other areas. The influence of 24-h urine salt excretion may differ between questions on this salt check sheet; some questions may be changed for this reason. In our opinion, concomitant use of the salt check sheet and urinary salt excretion may provide better and more practical information regarding salt reduction.

In conclusion, the total salt check-sheet scores significantly correlated with 24-h urinary salt excretion levels and can therefore be used to assess salt intake in local residents.

\section{CONFLICT OF INTEREST}

The authors declare no conflict of interest.

\section{ACKNOWLEDGEMENTS}

We thank Professor Kazufumi Yamane for his helpful advice on preparing the English version of the salt check sheet. This work was supported by a Grant-in-Aid for Scientific Research from the Japanese Society for the Promotion of Science (grant no. 25750067). 
1 Beaglehole R, Bonita R, Horton R, Adams C, Alleyne G, Asaria P, Baugh V, Bekedam H, Billo N, Casswell S, Cecchini M, Colagiuri R, Colagiuri S, Collins T, Ebrahim S, Engelgau M, Galea G, Gaziano T, Geneau R, Haines A, Hospedales J, Jha P, Keeling A, Leeder S, Lincoln P. McKee M, Mackay J, Magnusson R, Moodie R, Mwatsama M, Nishtar S, Norrving B, Patterson D, Piot P, Ralston J, Rani M, Reddy KS, Sassi F, Sheron N, Stuckler D, Suh I, Torode J, Varghese C, Watt J, Lancet NCDAG, Alliance NCD. Priority actions for the non-communicable disease crisis. Lancet 2011 ; 377: $1438-1447$.

2 Bibbins-Domingo K, Chertow GM, Coxson PG, Moran A, Lightwood JM, Pletcher MJ, Goldman L. Projected effect of dietary salt reductions on future cardiovascular disease. N Engl J Med 2010; 362: 590-599.

3 Ikeda $\mathrm{N}$, Inoue $\mathrm{M}$, Iso $\mathrm{H}$, Ikeda S, Satoh $\mathrm{T}$, Noda M, Mizoue $\mathrm{T}$, Imano H, Saito $\mathrm{E}$, Katanoda K, Sobue T, Tsugane S, Naghavi M, Ezzati M, Shibuya K. Adult mortality attributable to preventable risk factors for non-communicable diseases and injuries in Japan: a comparative risk assessment. PLoS Med 2012; 9: e1001160.

4 Anderson CA, Appel LJ, Okuda N, Brown IJ, Chan Q, Zhao L, Ueshima H, Kesteloot $H$, Miura K, Curb JD, Yoshita K, Elliott P, Yamamoto ME, Stamler J. Dietary sources of sodium in China, Japan, the United Kingdom, and the United States, women and men aged 40 to 59 years: the INTERMAP study. J Am Diet Assoc 2010; 110: $736-745$.

5 Toda A, Ishizaka Y, Tani M, Yamakado M. Current dietary salt intake of Japanese individuals assessed during health check-up. Hypertens Res 2015; 38: 163-168.

6 Asakura K, Uechi K, Sasaki Y, Masayasu S, Sasaki S. Estimation of sodium and potassium intakes assessed by two $24 \mathrm{~h}$ urine collections in healthy Japanese adults: a nationwide study. Br J Nutr 2014; 112: 1195-1205.

7 Okuda N, Nishi N, Ishikawa-Takata K, Yoshimura E, Horie S, Nakanishi T, Sato Y, Takimoto H. Understanding of sodium content labeled on food packages by Japanese people. Hypertens Res 2014; 37: 467-471.

8 Tsuchihashi T, Masuda K, Oniki H, Sakaki M, Arakawa K, Kameda W, Fukui H. Assessment of salt intake by using a simple check sheet in hypertensive patients. J Blood Press 2013; 20: 1239-1243.

9 Tanaka T, Okamura $\mathrm{T}$, Miura $\mathrm{K}$, Kadowaki $\mathrm{T}$, Ueshima $\mathrm{H}$, Nakagawa $\mathrm{H}$, Hashimoto $\mathrm{T}$. A simple method to estimate populational 24-h urinary sodium and potassium excretion using a casual urine specimen. J Hum Hypertens 2002; 16: 97-103.

10 Kobayashi S, Murakami K, Sasaki S, Okubo H, Hirota N, Notsu A, Fukui M, Date C. Comparison of relative validity of food group intakes estimated by comprehensive and brief-type self-administered diet history questionnaires against $16 \mathrm{~d}$ dietary records in Japanese adults. Public Health Nutr 2011; 14: 1200-1211.

11 Kobayashi S, Honda S, Murakami K, Sasaki S, Okubo H, Hirota N, Notsu A, Fukui M, Date $\mathrm{C}$. Both comprehensive and brief self-administered diet history questionnaires satisfactorily rank nutrient intakes in Japanese adults. J Epidemiol 2012; 22: 151-159.

12 Sakata S, Tsuchihashi T, Oniki H, Tominaga M, Arakawa K, Sakaki M, Kitazono T. Relationship between salt intake as estimated by a brief self-administered diet-history questionnaire (BDHQ) and 24-h urinary salt excretion in hypertensive patients. Hypertens Res 2015; 38: 560-563.

13 Tochikubo O, Uneda S, Kaneko Y. Simple portable device for sampling a whole day's urine and its application to hypertensive outpatients. Hypertension 1983; 5: 270-274.

14 Yasutake K, Horita N, Murata Y, Koyama S, Enjoji M, Tsuchihashi T. Estimated urinary salt excretion by a self-monitoring device is applicable to education of salt restriction. Hypertens Res 2015; 38: 143-148.

15 Yasutake K, Horita N, Umeki Y, Misumi Y, Murata Y, Kajiyama T, Ogimoto I, Tsuchihashi T, Enjoji M. Self-management of salt intake: clinical significance of urinary salt excretion estimated using a self-monitoring device. Hypertens Res 2016; 39: 127-132.

16 Ohta Y, Tsuchihashi T, Ueno M, Kajioka T, Onaka U, Tominaga M, Eto K. Relationship between the awareness of salt restriction and the actual salt intake in hypertensive patients. Hypertens Res 2004; 27: 243-246.

17 Sakaki M, Tsuchihashi T, Arakawa K, Fukui H, Kameda W, Tominaga M. Long-term variability of urinary salt excretion and blood pressure in hypertensive patients. Hypertens Res 2014; 37: 939-943.

18 Okubo H, Sasaki S, Hirota N, Notsu A, Todoriki H, Miura A, Fukui M, Date C. The influence of age and body mass index on relative accuracy of energy intake among Japanese adults. Public Health Nutr 2006; 9: 651-657.

19 Murakami K, Sasaki S, Takahashi Y, Uenishi K, Yamasaki M, Hayabuchi H, Goda T, Oka J, Baba K, Ohki K, Kohri T, Watanabe R, Sugiyama Y. Misreporting of dietary energy, protein, potassium and sodium in relation to body mass index in young Japanese women. Eur J Clin Nutr 2008; 62: 111-118.

20 Fukumoto A, Asakura K, Murakami K, Sasaki S, Okubo H, Hirota N, Notsu A, Todoriki H, Miura A, Fukui M, Date C. Within- and between-individual variation in energy and nutrient intake in Japanese adults: effect of age and sex differences on group size and number of records required for adequate dietary assessment. J Epidemiol 2013; 23: 178-186. 